\title{
A Comparative Study of the Sensory Structures Among Three Basal Hexapod Clades (Arthropoda: Collembola, Protura, Diplura) Using Scanning Electron Micrographs
}

\author{
Robert T. Allen ${ }^{1}$, Amanda Lawrence ${ }^{2}$, and Richard L. Brown ${ }^{1}$ \\ ${ }^{1}$ Mississippi Entomological Museum, Mississippi State University, Starkville, MS, USA. \\ ${ }^{2}$ Institute for Imaging \& Analytical Technologies, Mississippi State University, \\ Starkville MS, USA.
}

Collembola, Diplura, and Protura are basal orders of Hexapoda that are associated primarily with leaf litter and other decaying vegetative materials. These three clades historically have been considered monophyletic orders of insects, but some systematists recently have ranked them as classes independent of the class Insecta [1]. Collembola are unique in having a projecting furcula on the abdomen that is used for springing off a substrate. Diplura lack compound eyes and have abdominal styli. Protura lack compound eyes and antennae. The relationships among the three taxa have been called into question based primarily on DNA and physiological (sperm morphology) studies. A long term study of the external anatomy of these basal Hexapod orders using scanning electron microscopy has been initiated with the following objectives: 1) to determine if morphological characters not visible with light microscopy can be detected for use in identifying closely related taxa and 2) to identify "new" characters and/or character states useful in determining cladistic relationships. This poster presents some of the first results of the study concerning sensory structures in Collembola, Diplura, and Protura.

Samples were collected in 70\% ETOH then dehydrated in a graded ethanol series and chemically dried with HMDS (Hexamethyldisilazane). Insects were affixed to aluminum stubs with carbon tape and coated with Pt at $30 \mathrm{~nm}$ and examined with a JEOL JSM-6500F Scanning Electron Microscope at 5 kv.

The study has revealed considerable information on the sensory structures of these basal Hexapods. The number and diversity of sensory structures varies considerably among the major clades (Figures 1-6). Collembola have the fewest sensory structures, with most of these being trichoid sensilla concentrated on the terminal segment of the antenna (Figure 1). A unique form of pectinate sensillum with an unknown function is present on the abdominal furcula of Collembola (Figure 2). Diplura have both trichoid sensilla and trichobothria on their antennae (Figure 3). The trichobothria are ramose and arise from pits (Figure 4). The high number and diversity of sensilla on prolegs of Protura (Figures 5,6) may be related to the development and use of these appendages to compensate for loss of the antenna. The presence or absence of various sensilla is also variable within some clades. Some sensilla present in some taxa, e.g., the sensilla of the furcula of Collembola, may indicate strong apomorphic affinities (derived) useful in determining evolutionary relationships. Further study of significantly more taxa within each clade is warranted.

\section{References}

[1] D. Grimaldi and M. S. Engel. 2005. Evolution of the Insects. Cambridge University Press, New York. p. 1-755.

[2] The authors acknowledge support provided by the National Institute of Food and Agriculture, U.S. Department of Agriculture, under CRIS Project MIS-012040. 

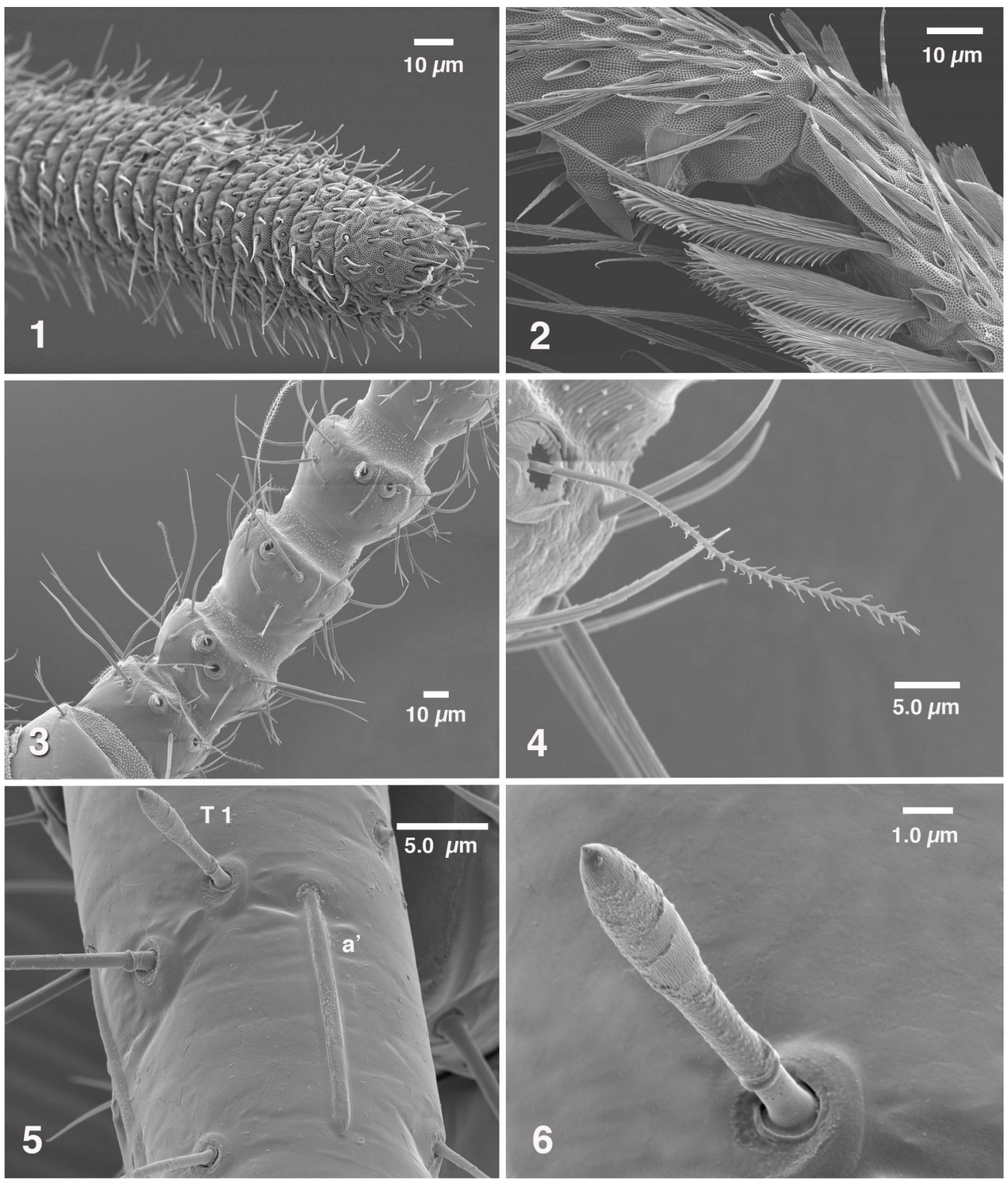

Figures 1-6. Sensilla of Hexapoda, 1, Collembola: terminal antennal segment. 2, Collembola: unknown type of pectinate sensilla on furcula. 3, Diplura: antennal segments 2-6. 4, Diplura: antennal trichobothria in pit. 5, Protura: protarsal sensilla t1 and a'. 6, Protura: protarsi sensilla t1. 